\title{
И.В. Брылина
}

\section{ОРГАНИЗАЦИОННАЯ СТРУКТУРА УНИВЕРСИТЕТА В УСЛОВИЯХ РЫНОЧНОЙ КОНКУРЕНЦИИ: ПОТЕНЦИАЛ АДАПТАЦИИ И РИСКИ}

\begin{abstract}
Исследуется адаптивный механизм университета в условиях рыночной экономики, позволяюший университетам, использующим более эффективную стратегию изменения своей организационной структуры, получать преимущества в условиях конкуренции.

Делается вывод, что природа университета подобна холдингу: гибкая, подвижная, открытая организационная структура университета в большей мере способна проявить и использовать свои адаптивные способности и механизмы.

Ключевые слова: исследовательский университет, организационная структура университета, механизм адаптации, конкуренция.
\end{abstract}

Во всем мире система высшего образования претерпевает изменения. В условиях развивающейся рыночной экономики работает адаптивный механизм университета. Б.-Р. Кларк в исследовании «Система высшего образования: академическая организация в кросс-национальной перспективе» [1] к числу вопросов организации и управления высшим образованием отнес фундаментальный адаптивный механизм университетов и академических систем. Этим механизмом, по мнению Б.-Р. Кларка, является способность прибавлять и удалять определенные области знания, не затрагивая других дисциплин. Адаптируемость строится на внутреннем многообразии объединенных, конгломератных структур. Э. Дюркгейм писал об университете как стихийном продукте эпохи Средних веков. Этот живой продукт настолько же узнаваем, насколько неповторим: «Это единство и разнообразие служат главным подтверждением того, что университет был стихийным продуктом средневековой жизни; ибо только живые существа могут, оставаясь самими собой, изворачиваться и приспосабливаться к различным обстоятельствам и условиям» [2. С. 163]. Это свойство и способность университета быть неповторимым (двух одинаковых университетов нет) и узнаваемым (во всех принимаемых ими обличьях) определены специфическим внутренним устройством университетов, институционализированным в специфичных системах высшего образования.

Б.-Р. Кларк, обращаясь к проблеме академической организации в кросснациональной перспективе, пишет об этом свойстве университетов («изворачиваться и приспосабливаться») как о зависимом от форм, присущих национальным системам высшего образования, и этими системами определяемом: «Одни формы способствуют адаптации к потребностям растущего знания, другие - сопротивляются им. Одним формам удается подготовиться к изменениям, и потому они, скорее всего, сохранятся и дальше. Другим это сделать не удается, и они служат примерами несостоятельных форм, стимулируя тем самым усилия по их отмене и развитию альтернатив» [1. С. 235]. Б.-Р. Кларк 
сравнивает национальные механизмы работы систем высшего образования, исходя из специфики горизонтальной и вертикальной дифференциаций, касающихся как внутрисистемных структур, так и межсистемных отношений и структур (секторы, слои, секции и их иерархия). Эти процессы горизонтальной и вертикальной дифференциации могут как содействовать трансформации систем высшего образования, так и противостоять им. Эта гипотеза блестяще иллюстрируется Б.-Р. Кларком в исследовании «Академическая власть в Италии» [3] и Ваном Граффом [4] на примере исследований, посвященных процессам перехода от кафедральной организации к организации по отделениям. Б.-Р. Кларк особо отмечает дисфункциональный и недемократичный характер кафедральной власти, когда пишет о том, какую роль в определении трансформаций играет кафедральная организационная структура или структура отделений операционного уровня. Автор видит ограничивающую адаптивную способность кафедр, ограниченный масштаб адаптаций как определяемый способностями и наклонностями руководителя кафедры. При этом ограничены возможности овладения дисциплиной. Академическая власть, проявляемая на уровне кафедральной организационной структуры, по природе своей дисфункциональна и недемократична. Основанием этой дисфункциональности и недемократичности является следующее обстоятельство. Растет число кафедр, работающих с огромным объемом знания, с широкой клиентурой. Одновременно с этим дробятся дисциплины, факультеты, университеты. Кафедры обретают громоздкость дисциплин и программ. Перегрузка эта служит выражением операционного развития, она ставит под сомнение легитимность такой организационной единицы, как кафедра, и делает очевидными дисфункциональность и недемократичность кафедральной власти.

Эту ситуацию для организационной системы высшего образования аналитики называют провальной. И считается, что именно очевидность этой провальности явилась основанием для отказа от кафедральной организационной системы уже в 60-70-е годы ХХ века. Однако от кафедральной системы ушли не все. К примеру, Швеция оставила кафедры, перейдя к организации по отделениям лишь частично, частично влияя на задачи, цели, проблематику проводимых на отделениях исследований.

Новыми учебно-исследовательскими единицами для французской системы высшего образования, начиная с событий 1968 года, явились unites d'Enseignement et de Recherche, пришедшие на смену традиционным факультетам. Эти учебно-исследовательские единицы включали отделения, пришедшие на смену кафедрам. В университетах Германии традиционные факультеты сменили секции, подобные тем, которые уже работали в системах образования Великобритании и Америки. В Германии на смену 960 институтам пришли 190 отделений. Эти изменения носили чрезвычайно радикальный характер. Однако эта радикальность была принята не всеми. Так, система общего образования не приняла идею отделений: колледжи (Оксфорда, Кембриджа), предоставляющие широкомасштабное и эффективное преддипломное образование, ориентированы на узкое представление о дисциплинарной специализации. Секции, именуемые отделениями, не могут обеспечить широкий спектр академических специальностей, существующих 
в университетах и постоянно расширяющихся. Современные университеты применяют матричные структуры (профессора и студенты заняты в центрах, решающих одни и те же проблемы в колледжах и отделениях, продолжительность обучения в которых составляет 4 года). Существует и «тройная» матрица (профессор занят в центре, на отделении и в секторе, объединены они по типу кластерной системы). Горизонтальная организационная система, принятая в университетском образовании, таит в себе большие возможности, поскольку с её помощью преодолевается ограниченность возможностей кафедр, что способствует созданию программ с большим объемом дисциплин. В целях исключения этой ограниченности необходимо большее количество надзорных структур и развитие таких структур высшего образования, в которых используется идея слоёв: в низшие уровни образовательных систем включаются программы, в которых синкретично объединена совокупность программ. Хотя самая распространенная форма устройства образовательной системы в высшем образовании - это один организационный слой, в пределах которого осуществляется подготовка к профессиональной или академической степени.

Американская модель, обладающая мощной адаптивной способностью, ориентирована на вертикальную дифференциацию. В этих условиях, как считает, к примеру, Б.-Р. Кларк, попасть во второй слой профессиональных школ, равно как и на последипломное обучение в таких науках, как гуманитарные и естественные, можно лишь закончив обучение в четырехлетнем колледже. Б.-Р. Кларком отмечена огромная значимость университетов в профессиональных движениях. В США первые школы последипломного обучения возникли в 70-е годы XIX века. И с этого десятилетия лучшие университеты были превращены в передний край профессионального самосознания. В них обучающихся готовили к профессиям, лишь начинавшим своё формирование: «К началу XIX века университеты стали бесспорной легитимационной инстанцией, поскольку ни одна новая профессия не могла считаться полноценной или научной, если для неё не существовало отдельного академического учебного плана. Они служили центрами философствования и пропаганды и прививали ученикам собственные ценности и цели. Принимая во внимание потенциал университета для преодоления фрустрации, было крайне важно, чтобы высшее образование было всеядным и даже неразборчивым, принимая все новые группы» [1. С. 239].

Помимо обозначенного первого слоя, американская структура образовательных систем опиралась на тот слой, который обеспечивал «расширение», - «всеядность» (в терминологии Б.-Р. Кларка, Р. Вейбе [5], Т. Бичара и М. Когана [6]). Б.-Р. Кларком названы и проанализированы следующие источники этой «всеядности».

Во-первых, организационная структура, разделяющая занимающихся специализированной профессиональной подготовкой и занимающихся общей подготовкой. Специализация при этом обозначена как ценность особой значимости.

Во-вторых, наличие множества административных должностей, включая должности декана последипломного обучения и декана профессиональной школы, занятых развитием обозначенного уровня. 
В-третьих, последипломный слой получал особую поддержку в области исследований. Эти источники «всеядности» являются внутриинституциональными источниками и проявляют себя в сфере конкуренции институтов.

В-четвертых, институты реагируют на специфику спроса «рынка труда», идут навстречу соображениям рациональности действий и программ и создают ряд школ такого профиля, как права, бизнеса, архитектуры, не ориентируясь при этом (в отличие от университетов «Лиги плюща») на соображения престижности: «Динамика институционального роста в более открытой и конкурентной институциональной среде... стала основным источником экспансионистских тенденций в углубленной подготовке» [1. С. 241].

И хотя многообразные национальные системы имеют много общего, они различны в интерпретации роли и значимости структуры слоёв. Студенты вузов Британии, в отличие от вузов Америки, делают акцент на преддипломной подготовке, не встречая помощи в работе на последипломном уровне. Уровень получения докторской степени в вузах Британии организационно менее обеспечен, в отличие от уровня получения начальной научной степени [7] .

Возникает вопрос: в чем заключены достоинства описанных организационных структур, применяемых в системах университетского образования Запада? Что дает обращение к потенциалу уровней и слоёв?

Обращаясь к возможностям уровней и слоёв, образовательная система способна обнаружить свою гибкость и адаптивность применительно к интенсивно трансформирующимся запросам социума. Аналитики футурологической направленности предвидят потребность в пяти уровнях и слоях и, соответственно, трансформацию системы ученых степеней. Сегодня американский университет способен предоставить возможность, помимо степени бакалавра, получить степень магистра (для этого необходим пяти- или шестилетний срок обучения), степень доктора (восемь или десять лет обучения). Также существует и университетская система профессиональных степеней (шесть или восемь лет обучения). Постдоковский диплом может быть получен в условиях пятого уровня, институционализированного сегодня не во всех областях научного знания.

Для организационных структур с одним слоем характерны трудности роста. Они вынуждают идти на системы циклического обучения (они предполагают множество программных уровней), что предусматривает сложности отбора. Это трудноосуществимая задача в условиях, когда декларируется идея массового образования и одновременно должна быть реализована идея избирательности, как и идея выбора между преподаванием и исследованием. Аналитики активно используют понятие «субспециальностей», обращаясь к исследованию ситуации, в которой рост слоёв способствует изменению границ и пределов организационного пространства, а синтез субспециальностей создает интегрированное основание последующей специализации. В описательной форме это выглядит следующим образом: «Более общий первый слой предлагает некоторую интеграцию в рамках дисциплин и между ними. Во французской системе первый цикл посвящен общей подготовке к дисциплине или профессии и представляет собой сочетание субспециальностей, составляющее интегрированную основу для более поздней специали- 
зации. Короче говоря, рост слоёв делает возможной не только большую специализацию, но и определенную консолидацию предметов внутри секций и между ними, оказывая тем самым некоторое противодействие разделению академических специальностей. Рост слоёв расширяет организационное пространство» [1. С. 243].

Диверсификация образовательных систем, их сложная организационная специфика способствуют трансформационным изменениям, как способствуют они и тому, чтобы быть использованными в системе разделения труда между институтами. Многотипные образовательные системы, приходящие на смену институциональным системам единого типа, в условиях конкуренции вузов и увеличивающегося многообразия функций проявляют большую адаптируемость. Если исключить это, университет утратит связь с рынками труда. Институционализация традиций, оборачиваясь косностью, мешает инициативам. Университет может потерять концентрацию ресурсов и способен утратить доверие к осуществляемой в границах университета традиционной деятельности.

Теоретики университетского образования связывают структурную упрощенность образовательных систем и утрату легитимности воедино. Эта утрата легитимности оказывается обусловленной неспособностью реагирования на ситуацию, связанную с изменениями. Уже в первое десятилетие XXI века университеты активно разрабатывают способы дифференциации, которые позволили бы этим институтам с многовековой историей миновать кризис. И хотя сегодня, как полагают аналитики, «за университет может сойти все что угодно», университетом является лишь «институциональный, всесторонний университет» - это всеобъемлющая структура. Аналитики уподобляют его холдингу. Университет подобного типа, полагает Б.-Р. Кларк, можно создать посредством дифференциации основных частей и установления формального равенства, что труднореализуемо в силу того, что более престижные части не принимают этого равенства. При этом «институциональная иерархия, как было отмечено ранее, варьируется от господства одного или двух университетов или относительно небольшого элитного сектора до умеренного, но открытого институционального ранжирования и даже незначительных различий в престижности или полного отсутствия таковых» [1. С. 244].

Что касается системы университетского образования США, в ней доминирующим фактором являются исследовательские университеты. Для них характерна интенсивная институциональная мобильность, проявившая свою масштабность в 80-е гг. XX века, когда университеты Стэнфорда и Калифорнии изменили свой статус. Что же касается общей тенденции, она заключена в том, что секции, слои, секторы и иерархия простого устройства, как правило, направлены в сторону сдерживания стихийных изменений, в то время как сложность устройства интенсифицирует изменения подобного рода. И в условиях этой сложности национальные образовательные системы идут на синтез ряда организационных измерений, - секций, слоёв, секторов, иерархических особенностей.

Вернемся на столетие назад и обратимся к явлению, значимость которого трудно переоценить. Речь идет о том, что в совокупности колледжей, многие 
из которых существовали на протяжении двух столетий, появился университет. Модель американского университета (как пример вертикальной дифференциации) была ориентирована на проведение исследований и фундаментальное образование. Эта модель возникла по образу и подобию университетов Германии. Колледжи в системе высшего образования Америки сосуществовали с возникающими университетами и сохранили свое статусное предназначение в силу ряда причин. Одной из них явилась причина, отмеченная Бен-Дэвидом: общественное мнение видело в колледжах форму институционализированную, а тормозом реформы системы образования явился тот факт, что «не было никаких центральных организаций, чтобы проводить изменения и оплачивать за государственный счет услуги, которые могли не пользоваться спросом» [8. С. 81].

Не утрачивает своей актуальности проблема доступности университетского образования. Для стран Европы эта проблема превратилась в политическую уже во второй половине ХХ века, когда отбор, по сути, приобрел форму отказа в доступе представителям средних и низших классов. Двух- или многослойная структура университетской образовательной системы позволяет обеспечить широкий доступ к образованию. В то же время высшая степень избирательности присуща верхним слоям (таковы принципы отбора в школах права и школах архитектуры). Систему высшего образования, таким образом, характеризуют и открытость, и ограниченность [9].

Что касается стран, в образовательных системах которых преобладают однослойные структуры, стоит отметить,что эта однослойность вызывает перегрузку структуры, ведет к утрате концентрации системы и рынка труда, к возникновению глубокого разрыва в системе ожидания студентов (а эти ожидания формировались в ходе долгой истории занятости выпускников в элитных сферах и того, что предлагает жизнь: незначительное число вакантных должностей элитарного уровня и резкого возрастания объемов предложений профессионалов в границах рынка труда). Гамильтон и Райт в исследовании «Coming of Age» следующим образом описывают эту ситуацию, когда перспектива элитарной занятости исчезла практически полностью: «Каналы обучения нередко принимали в пять-десять раз больше людей, рассчитывающих на высокое вознаграждение, чем требовали традиционные рынки. Потребность в учителях средней школы отпала после того, как в результате роста высшего образования, имевшиеся вакансии были заполнены первым и вторым поколением учителей, поступивших на постоянную гражданскую службу; точно так же чиновничий аппарат не может расти бесконечно, принимая на службу все новых выпускников университета, даже если страны, вроде Италии или Мексики, могут иметь давнюю традицию подобного подхода к смягчению конфликта за счет роста правительства; и некоторые другие профессии также сталкиваются с угрозой безработицы для квалифицированных кадров и сокращения заработной платы вследствие слишком быстрого роста предложения профессионалов на рынке труда» [10. С. 341].

Эта ситуация, по мнению аналитиков и теоретиков университетского образования (Кларка, Бэн-Дэвида, Гамильтона, Райта), заставляет идти на процесс все более выраженной интенсивной вертикальной дифференциации в границах вузовских структур через отбор на уже имеющихся уровнях обра- 
зовательных систем. Б.-Р. Кларку принадлежит плодотворная эвристическая гипотеза, смысл которой заключен в следующем: различия в структурных связях слоёв ведут к поддержке исследований, в которых выражена элитарная функция вуза. Хотя, по мнению Б.-Р. Кларка, есть все основания видеть очевидность условий, в которых государство вынуждено разделять исследование и преподавание. «Исследования, - считает Б.-Р. Кларк, - переживают сложные времена, когда невероятно разросшиеся общеобразовательные университеты отдают их на откуп преподавателям, которым приходится нести тяжелое бремя массового обучения и консультирования. Те, кого больше всего привлекает исследовательская деятельность, сталкиваются с ситуацией, когда они вынуждены тратить свои силы на другие вещи, и потому они стремятся перейти во внешние исследовательские организации, побуждая государство отделять исследования от преподавания в форме отдельной структуры научно-исследовательских институтов» [1. С. 72].

В условиях работы образовательных систем, имеющих в своей структуре два и более слоёв, существует возможность сохранения исследований в последипломный период. Эта возможность ориентирована на идею: только в условиях сохранения исследовательского блока можно связать исследования и подготовку талантливых специалистов, исключив для этой категории преддипломное обучение и сохраняя для этой категории обучающихся возможность обучения в своем институте. Именно об этой ситуации в работе «Центры обучения» и пишет Бэн-Дэвид, справедливо, на наш взгляд, полагая, что исследование и преподавание сосредоточено сегодня в различных слоях и является реализацией различных функций систем высшего образования. И лишь объединение этих функций - путь к реализации идей общего образования в университетах. В условиях одного слоя, к тому же предельно специализированного, реализация идеи качественного общего образования невозможна.

Организационная система американских университетов достаточно гибка, подвижна и в силу этого в полной мере способна обнаружить свои адаптивные способности. Она способна обеспечивать возможность доступа к институту образования. Также она обеспечивает (именно в силу своей гибкости и адаптивности) связь с рынком труда. Именно организационная система способна обеспечить поддержку таких элитарных функций университетского образования, как функция исследовательская. Таковы достоинства этой системы.

Почему же в таком случае европейский университет в XXI веке не принял для себя возможность развития по американскому типу и образцу? На наш взгляд, огромную роль играет традиция, как это было с колледжами в Америке во времена появления университета. Форма колледжей, как отмечает ряд исследователей, в массовом сознании была глубоко институционализирована, и именно в силу этого колледж с появлением университета не исчез. В 60-70-е годы ХХ столетия существовала идея (её автором был Мартин Э. Троу), смысл которой заключался в том, что все системы университетского образования будут иметь единую траекторию эволюции, в ходе которой будет осуществляться переход от элитарных форм к формам массовым. Аналитики полагали, что демократизация, экономический рост, расширение 
знания, политическое руководство образованием - все это вехи эволюционного развития университетов, динамичных процессов роста, проявляющих себя в сфере высшего образования Америки. Мартин Э. Троу полагал, что европейские модели университетского образования будут ориентироваться на такие институциональные трансформации, которые характерны американским моделям, и произойдет сближение национальных образовательных систем. Ситуация, однако, изменилась уже в 1973 году, когда стало ясно, что в выводе о сближении национальных систем Европы и Америки не был учтен фактор реакции и, напротив, переоценен спрос. Это признал и сам Мартин Э. Троу, писавший в работе «Элиты и массы высшего образования» об ошибочности своего вывода, сделанного в «Проблемах перехода элитного образования в массовое»: «Один из уроков 1970-х годов состоял в том, что существующие формы и устойчивые представления среднего и высшего образования во многом определили ограничение роста до 10-25 \% от возрастной группы или его безудержное продолжение до 40-50 \% или более. Способность и готовность к продолжению роста заметно различались в различных национальных системах. На самом деле, сторона «предложения», состоявшая из институтов и их ответных действий, во многом определяла, какие тенденции и потребности будут эффективными. Существующие системы Франции, Западной Германии, Италии и других стран определили потребности по-разному и потому не обязательно должны отвечать на внешне общие потребности точно так же, как и США» [11. C. 273].

В заключение можно сделать вывод, что «всесторонний университет», основанный на вертикальной дифференциации образования, является более эффективным (гибким, подвижным и открытым). По своей природе он подобен корпорации, основными принципами которой являются принципы дифференциации и формального равенства и которая имеет лучше отлаженный механизм и больший потенциал адаптации над теми университетами, которые основаны на горизонтальном способе организации (кафедрального типа). Они утрачивают жизнеспособность и являются менее эффективными в контексте современной рыночной конкуренции.

Вертикальная модель организационной структуры университета является наиболее конкурентоспособной, так как обеспечивает большую возможность взаимодействия двух рынков: рынка образования и рынка труда.

Другим значимым уроком XX века стало и то, что произошла переоценка спроса на образование и недооценка трансформации университета. Это явилось как основанием, так и следствием того, почему европейские образовательные системы избрали иной путь в сравнении с тем, которым пошли университеты Америки, что, несмотря на сходные условия, их реализация может оказаться различной в разных странах.

\section{Литература}

1. Кларк Б.P. Система высшего образования: академическая организация в кросснациональной перспективе. М.: Изд. Дом. ВШЭ, 2011. 360 с.

2. Durkheim E. The Evolution of Educational Thought - Lectures on the formation and development of secondary education in France. Taylor \& Francis, 2005. 388 p.

3. Clark B.-R. Academic Power in Italy: Bureaucracy and Oligarchy in a National University System. Chicago: University of Chicago Press, 1977. 277 p. 
4. Van de Graaf J.H., Clarck B.-R., Furth D., Goldschmidt D., and Wheeler D. Academic Power: Patterns of Authority in Seven National Systems. New York: Praeger, 1978. 302 p.

5. Wiebe R. The search for order, 1877-1920. New York: Hill and Wang, 1967. 352 p.

6. Becher T., and Kogan M. Process and Structures in Higher Education. London: Routledge, 1992. 209 p.

7. Тейлор Джс. Причины перемен в высшем образовании Англии: стратегия развития научно-исследовательской работы в английских университетах // Вопросы государственного и муниципального управления. 2009. № 4. С. 136-146.

8. Ben-David J. Centers of Learning: Britain, France, Germany, United States. New Bruswick: Transaction Publishers, 1992. XV, 208 p.

9. Brylina I.V., Brylin V.I., Khaldeeva M.A., Lutoshkina O.S. Transforming into an entrepreneurial university: adaptation and risks [Electronic resources] 11th International Technology, Education and Development Conference (INTED2017), 6-8 March, 2017, Valencia, Spain: proceedings. Valencia: $\quad$ IATED, 2017. P. 10050-10056. Режим доступа: https://library.iated.org/view/BRYLINA2017TRA (дата обращения 11.05.2017)

10. Hamilton R., and Wright J. Coming of Age - A Comparison of the United States and the Federal Republic of Germany. Zeitschrift fur Soziologie, 1975. 275 p.

11. Trow M. Reflections on the Transition from Elite to Mass to Universal Access: Forms and Phases of Higher Education in Modern Societies since World War II. Springer International Handbooks of Education, 2007. Vol. 18. P. 243-280.

Irina V. Brylina. National Research Tomsk Polytechnic University (Tomsk, Russian Federation) E-mail: ibrylina@yandex.ru

DOI: $10.17223 / 1998863 X / 39 / 9$

ORGANIZATIONAL STRUCTURE OF UNIVERSITY ON THE COMPETITVE MARKET: ADAPTATION POTENTIAL AND RISKS

Key words: Research University, organizational structure of university, adaptation mechanism, competition

The article touches upon the issue of adaptation mechanism of university organizational structures under the conditions of market economy. The potential of adaptation mechanism is revealed. The authors propose the idea that adaptability is based on the internal diversity of combined conglomerate structures. The issue of adaptation is considered from the perspective of national higher education systems. Transnational perspective of organizational structures transformation in higher education is set out.

The author clarifies the specificity of horizontal and vertical differentiation, both related to intrastructures and interconnection structures and relations (sectors, layers, sections and their hierarchy). It is shown that the processes of horizontal and vertical differentiation promote the transformation of higher education systems on the one hand and resist these transformations on the other hand. The author provides examples why certain bases and principles of organizational structures in higher education (for example, organizational structure, where a department plays the role of a base) lose its vitality and start to transform. A comparative analysis of horizontal and vertical differentiation of higher education systems is carried out; the potential of differentiation in the dynamics of institutional growth in the competitive environment of higher education is revealed.

In the conclusion of this article the author refers to the concept of «institutional comprehensive university». It is stated that the nature of such university is similar to the nature of a holding company; the principles on which the integral construction of a «comprehensive university» is based present the principles of differentiation and formal equality. As it is shown in the article, these principles may be hardly connected.

It is concluded that flexible, mobile, open organizational system of higher education may show and use its abilities and adaptive mechanisms to a greater extent and degree. It provides a great opportunity of access to education, as well as the connection with labor market. In addition, this type of system provides support for the elite function of university education - for research function.

\section{References}

1. Clark, B.-R. (2011) Sistema vysshego obrazovaniya: akademicheskaya organizatsiya v krossnatsional'noy perspective [The Higher Education System: Academic Organization in Cross-National Perspective]. Translated from English by A. Smirnov. Moscow: HSE. 
2. Durkheim, E. (2005) The Evolution of Educational Thought - Lectures on the formation and development of secondary education in France. Taylor \& Francis.

3. Clark, B.-R. (1977) Academic Power in Italy: Bureaucracy and Oligarchy in a National University System. Chicago: University of Chicago Press.

4. Van de Graaf, J.H., Clark, B.-R., Furth, D., Goldschmidt, D., \& Wheeler, D. (1978) Academic Power: Patterns of Authority in Seven National Systems. New York: Praeger.

5. Wiebe, R. (1967) The Search for Order, 1877-1920. New York: Hill and Wang.

6. Becher, T., \& Kogan, M. (1992) Process and Structures in Higher Education. London: Routledge.

7. Taylor, J. (2009) Prichiny peremen v vysshem obrazovanii Anglii: strategiya razvitiya nauchno-issledovatel'skoy raboty $\mathrm{v}$ angliyskikh universitetakh [Causes of change in higher education in England: a strategy for the development of research work in English universities]. Voprosy gosudarstvennogo i munitsipal'nogo upravleniya - Public Administration Issues. 4. pp. 136-146.

8. Ben-David, J. (1992) Centers of Learning: Britain, France, Germany, United States. New Bruswick: Transaction Publishers.

9. Brylina, I.V., Brylin, V.I., Khaldeeva, M.A. \& Lutoshkina, O.S. (2017) Transforming into an entrepreneurial university: adaptation and risks. The Eleventh International Technology, Education and Development Conference (INTED2017). Valencia, Spain. March 6-8, 2017. pp. 10050-10056. [Online] Available from: https://library.iated.org/view/BRYLINA2017TRA. (Accessed: 11th May 2017).

10. Hamilton, R. \& Wright, J. (1975) Coming of Age - A Comparison of the United States and the Federal Republic of Germany. Zeitschrift fur Soziologie. 4(4). pp. 335-349.

11. Trow, M. (2007) Reflections on the Transition from Elite to Mass to Universal Access: Forms and Phases of Higher Education in Modern Societies since World War II. Springer International Handbooks of Education. 18. pp. 243-280. 